\title{
Osteosíntesis asistida por artroscopia de las fracturas de escafoides
}

\author{
C. Martínez Andrade \\ Servicio de Traumatología y Cirugía Ortopédica. Hospital Dos de Maig. Barcelona. España.
}

\begin{abstract}
Resumen: El tratamiento de las fracturas de escafoides ha evolucionado gracias a la introducción de las técnicas quirúrgicas mínimamente invasivas. La osteosíntesis percutánea es, en la actualidad, una alternativa al tratamiento conservador en las fracturas no desplazadas, especialmente en pacientes jóvenes y atletas, con buenos resultados y escasas complicaciones. Sin embargo, presenta limitaciones para el diagnóstico de lesiones de partes blandas que se asocian a estas fracturas. La utilización de la artroscopia de muñeca asociada a la cirugía percutánea, permite un correcto diagnóstico y tratamiento de estas lesiones asociadas y posibilita una correcta manipulación y reducción de la fractura sin necesidad de cirugía abierta disminuyendo así las complicaciones y mejorando el pronóstico de estas fracturas.
\end{abstract}

Palabras clave: Escafoides, fijación de fractura, artroscopia muñeca, técnica quirúrgica, percutáneo.

\begin{abstract}
Treatment of scaphoid fractures has evolved with the introduction of minimally invasive surgical techniques. The percutaneous osteosynthesis is now an alternative to conservative treatment in non-displaced fractures, especially in younger patients and athletes, with good results and few complications. However, has limitations in the diagnosis of associated soft tissue injuries with these fractures. Utilizing the associated wrist arthroscopy percutaneous surgery allows a correct diagnosis and treatment of these lesions and allows proper handling and fracture reduction without requiring open surgery thereby reducing complications and improving the outcome of these fractures.
\end{abstract}

Key words: Scaphoid, fracture fixation, wrist arthroscopy, surgical technique, percutaneous.

El tratamiento de las fracturas de escafoides ha evolucionado gracias a la introducción de las técnicas quirúrgicas mínimamente invasivas.

La osteosíntesis con tornillo de compresión por vía percutánea es, en la actualidad, una alternativa al tratamiento conservador en aquellas fracturas no desplazadas o mínimamente desplazadas. No existe evidencia de un mejor resultado del tratamiento quirúrgico ${ }^{1-3}$, e incluso se asocia a una tasa mayor de complicaciones, la osteosíntesis en las fracturas de escafoides, permite acortar el período de inmovilización e iniciar la rehabilitación de forma precoz, lo que conlleva una reincorporación más rápida a las actividades cotidianas si bien ${ }^{4-6}$.

Por el contrario, no existen dudas respecto a la indicación del tratamiento quirúrgico en las fracturas desplazadas de escafoides, en las que afectan al polo proximal o en aquellas que se asocian a luxaciones del carpo, siendo la reducción abierta y la fijación interna el tratamiento propuesto para todas ellas ${ }^{7-10}$.

Se han descrito complicaciones relacionadas con el tratamiento quirúrgico "abierto", en la mayoría relacionadas con el abordaje: cicatrices hiperálgicas, rigidez, inestabilidad secundaria a lesión ligamentosa, necrosis de polo proximal, etc. ${ }^{1,1 !}$.
En los últimos años, se han comunicado excelentes resultados con el uso combinado de las técnicas de cirugía percutánea y la artroscopia de muñeca para el tratamiento tanto de las fracturas agudas, como de casos seleccionados de retardo de consolidación y pseudoartrosis de escafoides ${ }^{10,12-15}$. Ventajas de la osteosíntesis asistida por artroscopia

\section{VENTAJAS DE LA OSTEOSÍNTESIS ASISTIDA POR ARTROSCOPIA}

Entre las ventajas que nos ofrece la utilización de la artroscopia de muñeca cabe destacar tres:

I. Valoración precisa del grado de desplazamiento, de la inestabilidad de la fractura y de la calidad de la reducción obtenida.
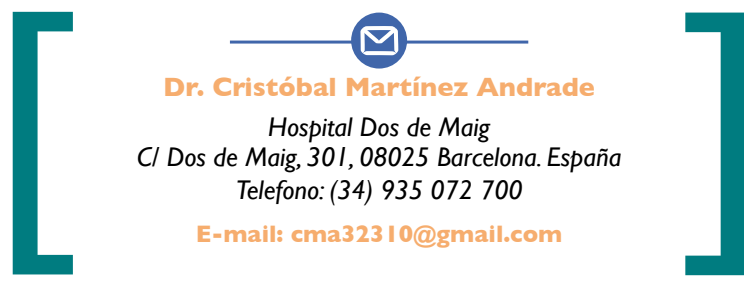
2. Diagnóstico y tratamiento de lesiones ligamentosas asociadas.

3. Visualización directa del punto de entrada del tornillo en el polo proximal

\section{VALORACION DEL DESPLAZAMIENTO Y LA INESTABILIDAD DE LA FRACTURA DE ESCAFOIDES}

El desplazamiento de los fragmentos es un factor de riesgo para la evolución de las fracturas de escafoides hacia la falta de consolidación. Con frecuencia, los términos desplazamiento e inestabilidad se confunden cuando nos referimos a las fracturas de cintura de escafoides. Así, debemos diferenciar entre una fractura de escafoides desplazada, que es aquella cuyos fragmentos presentan o bien un desplazamiento de más de $1 \mathrm{~mm}$ en cualquiera de los planos o bien una angulación entre ellos en el plano sagital superior a $15^{\circ}$, y una fractura inestable, que es aquella cuyos fragmentos se pueden movilizar mediante una ligera manipulación 16, 17. Todas las fracturas desplazadas son inestables, pero también lo son un porcentaje de las fracturas no desplazadas, lo cual podría justificar el fracaso del tratamiento conservador en este tipo de fracturas. La localización del trazo de fractura distal al vértice de la cresta dorsal del escafoides o la presencia de conminución se han relacionado con la inestabilidad ${ }^{17}$. Tanto la radiología convencional como la tomografía axial computerizada (TAC) son deficitarias para el diagnóstico, ya sea del grado de desplazamiento como de la inestabilidad de estas fracturas, con una sensibilidad y especificidad muy inferiores a las que ofrece la artroscopia de muñeca que se ha mostrado como el patrón oro a la hora de definir las características de estas fracturas y que, por lo tanto, nos permite optimizar el tratamiento eligiendo la mejor opción terapéutica ${ }^{18}$ (2) Figura I.

\section{VALORACION DE LESIONES LIGAMENTOSAS ASOCIADAS.}

Tradicionalmente, se ha considerado infrecuente la asociación entre lesiones ligamentosas intrínsecas o extrínsecas del carpo y las fracturas de escafoides no desplazadas o mínimamente desplazadas, limitando esta asociación a las fracturas-luxaciones provocadas por traumatismos de alta energía ${ }^{19,20}$. Esto puede explicarse por la visión limitada que ofrece el abordaje percutáneo por el que se accede a este tipo de fracturas.

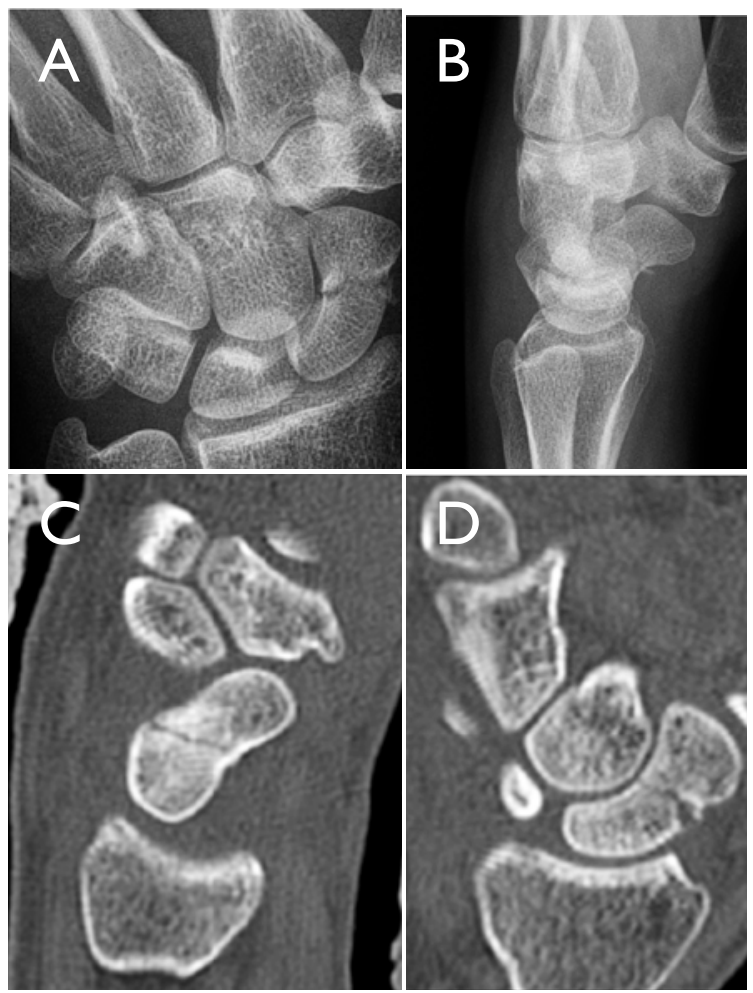

Figura I. Fractura de cintura de escafoides con conminución de margen radial y aparentemente mínimo desplazamiento. A. B. Radiología simple. C. D. TAC.

En los últimos años, son varios los trabajos que ponen de manifiesto un alto grado de asociación entre lesiones ligamentosas y fracturas de escafoides objetivadas al utilizar la artroscopia de muñeca de forma conjunta a la osteosíntesis percutánea, sin que exista relación entre la presencia ni la gravedad de las lesiones ligamentosas y el grado de desplazamiento de la fractura de escafoides ${ }^{21-23}$ @ Figura 2. Shih y cols. ${ }^{21}$ reportan un 13\% de lesiones del ligamento escafolunar y un $27 \%$ de lesiones del ligamento lunopiramidal en su serie. Jorgsholm y cols. ${ }^{23}$ en su estudio sobre 41 fracturas (26 de ellas no desplazadas) reporta un $71 \%$ de lesiones del ligamento escafolunar, un 20\% de lesiones del ligamento lunopiramidal y un $27 \%$ de roturas del fibrocartílago triangular.

El correcto diagnóstico y tratamiento de estas lesiones ligamentosas cobra mayor relevancia en la actualidad, ya que la movilización precoz que se suele aplicar a una osteosíntesis estable, empeora la cicatrización de estas lesiones y de esta forma puede comprometer el pronóstico y resultado de estas fracturas, en aparencia "benignas". 


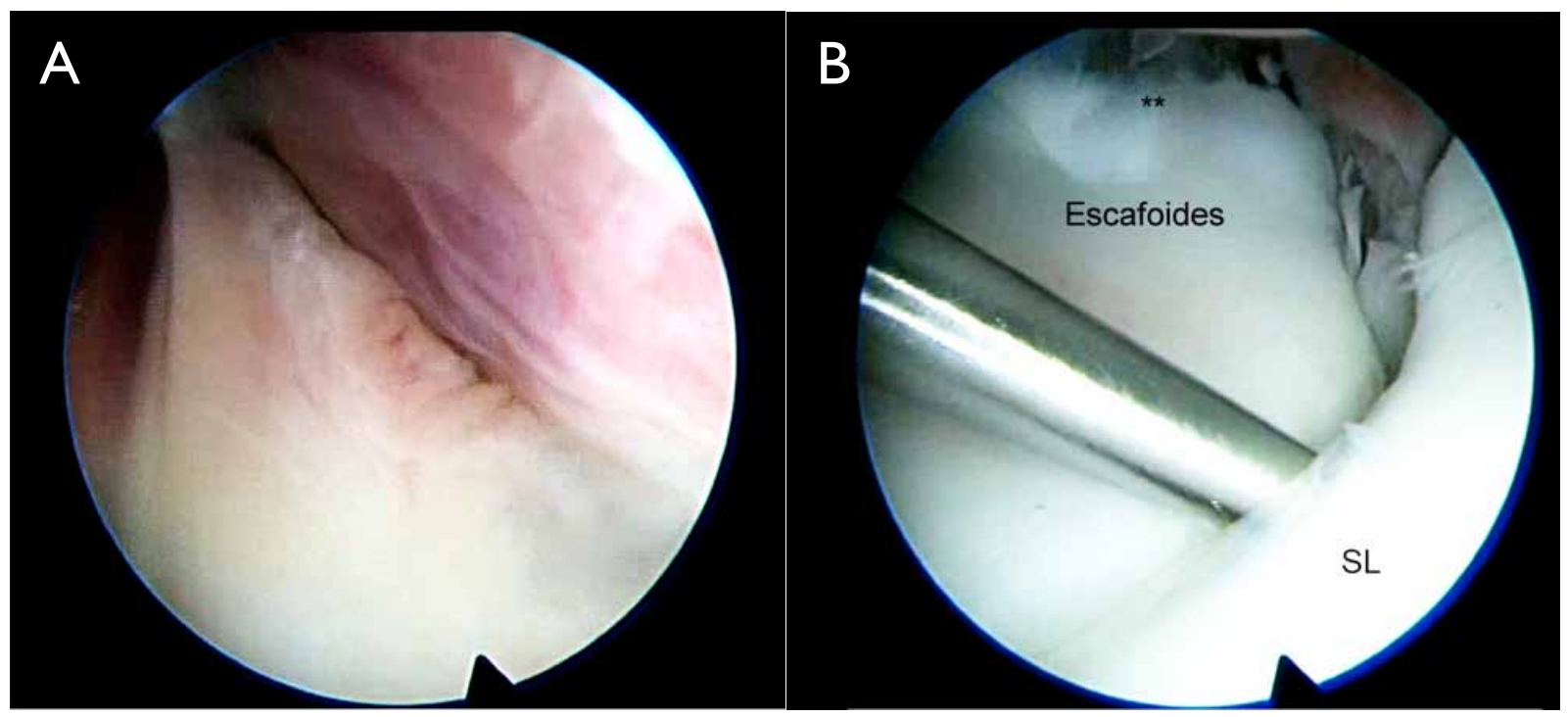

Figura 2. Lesión grado 2 de ligamento escafolunar ${ }^{25}$ asociada a fractura de escafoides de muñeca derecha. A.Visión desde portal 6R: porción dorsal del ligamento hemorrágica sin solución de continuidad. B. Visión desde portal mediocarpiano cubital: introducción de gancho palpador en espacio escafolunar con visión de fractura de cintura de escafoides sin desplazamiento **. SL: semilunar.

\section{VISUALIZACIÓN DIRECTA DEL PUNTO DE ENTRADA DEL TORNILLO EN EL POLO PROXIMAL}

Bajo control artroscópico, se puede localizar de manera exacta el punto de entrada en el polo proximal, que se localiza en la inserción del ligamento escafolunar en el escafoides. Se consigue así que la longitud del tornillo sea la máxima y que quede perfectamente colocado en el eje del hueso. Estos dos factores se han asociado con un mejor resultado en el tratamiento de las fracturas de escafoides ${ }^{24}$.

Del mismo modo, se puede comprobar que el tornillo queda enterrado dentro del hueso, con lo que se evitaría ulceras condrales en el radio por una longitud excesiva, muy difícil de valorar en controles radiológi$\cos$ (2) Figura 3.

\section{TÉCNICA QUIRÚRGICA}

Desde su primera descripción ${ }^{12}$, son diferentes las técnicas utilizadas en la osteosíntesis asistida por artroscopia de fracturas de cintura de escafoides. Si bien existe alguna serie en la que dicha osteosíntesis se realiza mediante tornillo de compresión de colocación retrógrado por abordaje palmar ${ }^{14}$, es el abordaje dorsal descrito por Slade en $2001^{10}$ el utilizado con mayor frecuencia para el tratamiento de estas fracturas. Más tarde,
Geissler ${ }^{15}$ modifica esta técnica introduciendo la visión directa artroscópica para la ubicación del punto de entrada de la aguja guía del tornillo.

Con el paciente en decúbito supino y tras inflado de torniquete neumático, se realiza una tracción de 5-6 $\mathrm{Kg}$ al cénit de la extremidad afecta, es importante que el sistema de tracción que se utilice permita la flexión de la muñeca para la correcta colocación de la aguja guía. Se introduce la óptica por el portal 3/4 para valorar la existencia de lesiones de partes blandas asociadas Figura 4. Posteriormente, se traslada la visión al portal $6 R$ y, tras flexionar la muñeca, se procede a identificar el punto de entrada del tornillo, que se ubicaría en la inserción del ligamento escafolunar en el polo proximal del escafoides. No es infrecuente que tejido sinovial dorsal hiperémico pueda dificultar la correcta visualización de dicho punto por lo que se debe realizar desbridamiento con sinoviotomo si es preciso. Tras identificar el punto óptimo de entrada, se introduce por el portal 3/4 un abbocath del calibre 14 y se insinúa en la cortical dorsal del escafoides (4) Figura 5. A continuación, se realiza la comprobación radiológica del correcto punto de entrada y se procede a la introducción de la aguja de Kirschner a través del abbocath, siguiendo el eje mayor del escafoides. Para este paso son de gran utilidad los sistemas de tracción que nos permitan colocar en posición horizontal la extremidad.

Una vez realizada la medición de la longitud del tornillo, se avanza la aguja guía hacia volar, para permitir la 


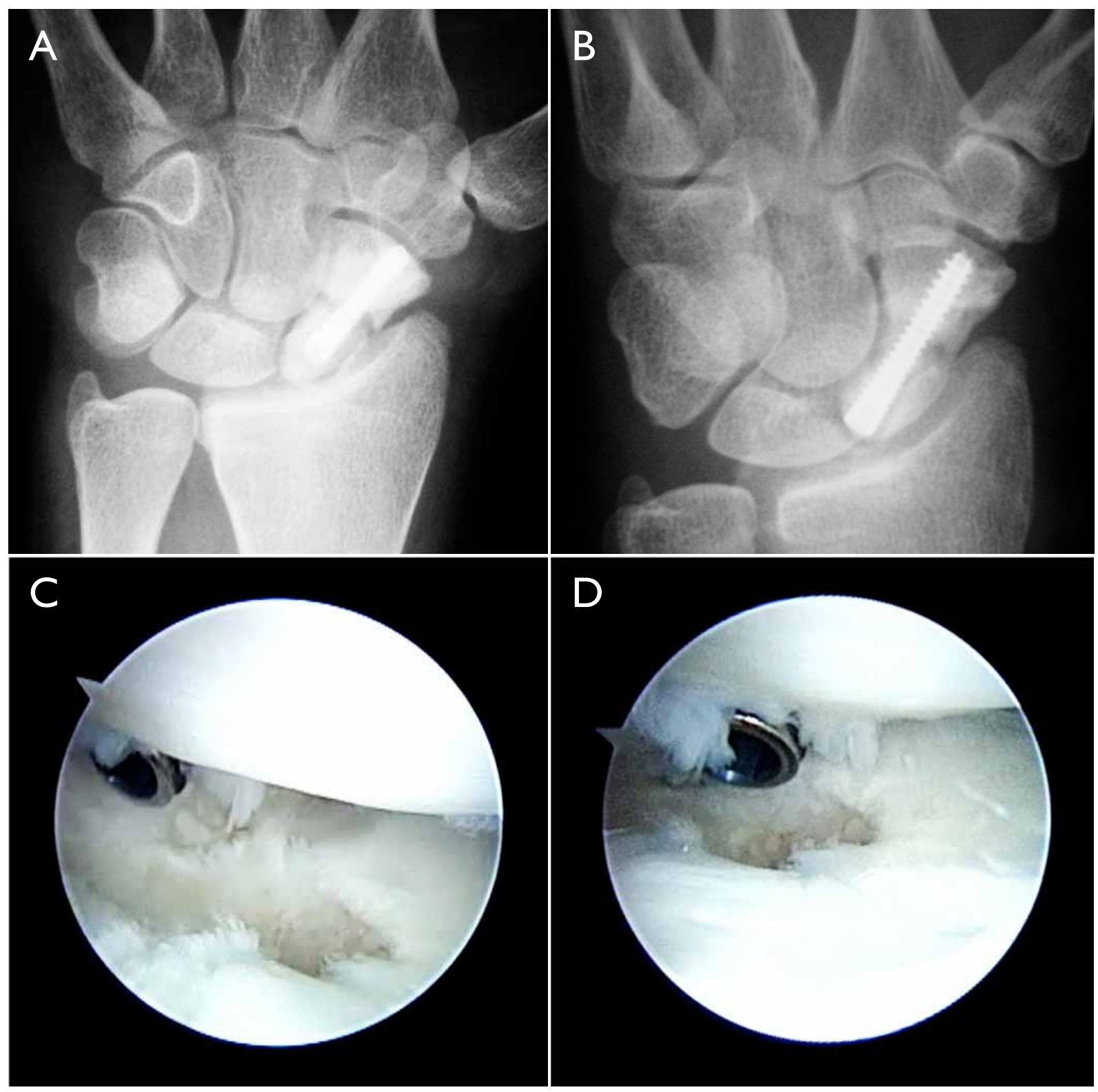

Figura 3. A y B. Imagen radiológica de paciente en el que se había realizado síntesis percutánea, que presentaba dolor con la extensión de la muñeca. C y D. Visión desde el portal 6R de úlcera condral en dorso de radio, por protrusión (oculta en la radiografía) del tornillo de osteosíntesis.

extensión de la muñeca y minimizar el riesgo de rotura con maniobras ulteriores, en este momento se procede a valorar artroscópicamente la reducción obtenida, para ello se utilizan los portales mediocarpianos radial (MCR) y cubital (MCC) (2) Figura 6.

Si no se ha conseguido una reducción aceptable se procede a retirar la aguja guía dejándola en el fragmento distal, seguidamente, se procede a la reducción de los fragmentos ya sea de forma indirecta, mediante manipulación e hiperextensión de la muñeca o utilizando agujas de Kirschner a modo de joysticks para movilizar los fragmentos, o bien de forma directa, manipulando el fragmento distal con un gancho palpador o con un periostotomo que se introducirán por el portal MCR (a) Figura 7.

Tras conseguir la reducción, avanzamos la aguja guía hacia el polo proximal y la recuperamos por el portal 3/4. Tras ampliar mínimamente el portal 3/4 mediante disección roma y proteger las partes blandas, se realiza el brocado y posterior colocación del tornillo canulado. 


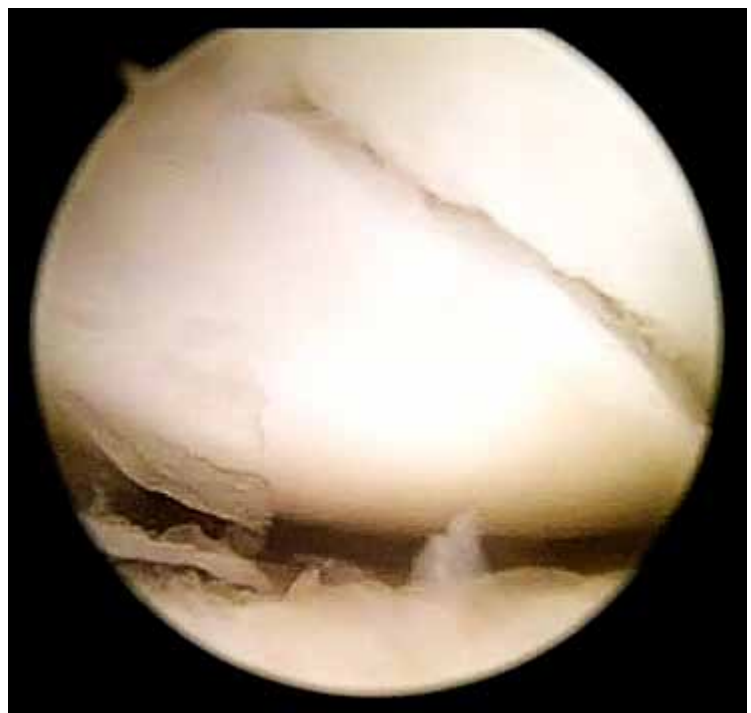

Figura 4. Fractura de polo proximal de escafoides desde portal $3 / 4$.

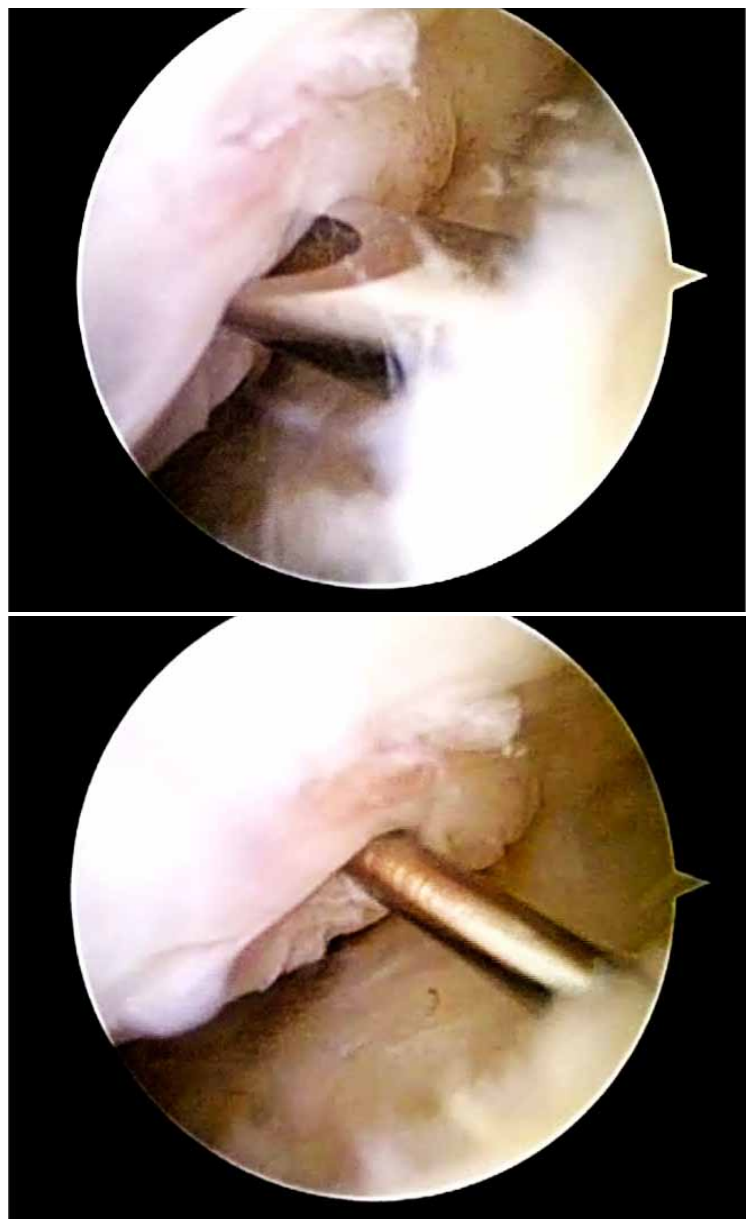

Figura 5. Identificación del punto de entrada del tornillo con visión en portal 6R. A. Localización del punto de entrada con abbocath. B. Aguja guía colocada.

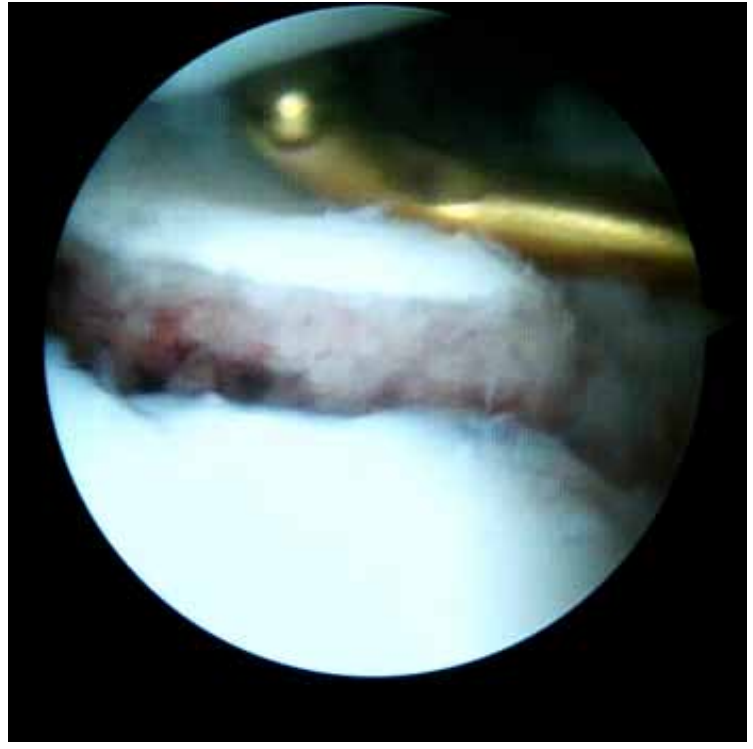

Figura 6. Visión de la fractura de escafoides, desde el portal mediocarpiano cubital, nótese el desplazamiento interfragmentario. Imagen correspondiente al caso de la figura I en el que no se apreciaba desplazamiento.

Una vez realizada la osteosíntesis, se procede a un control artroscópico. Desde los portales radiocarpianos, nos cercioramos que el tornillo está introducido en su totalidad en el escafoides, sin que protruya en el polo proximal, para evitar la posibilidad de provocar lesiones cartilaginosas en la faceta escafoidea del radio y, por último, desde los portales mediocarpianos, se realiza una comprobación final de la reducción de la fractura (c) Figura 8.

Se deja inmovilizada la muñeca con una férula volar por 10-12 días, seguida de una rehabilitación progresiva, pudiendo estar hábil para las actividades regulares y deportivas en 3-4 semanas, salvo que existan lesiones asociadas.

\section{RESULTADOS Y COMPLICACIONES}

Si bien las series son pequeñas, los resultados publicados son buenos, con una tasa de consolidación entre el 90-100\% de los casos ${ }^{21,22}$. Slade y Guillo ${ }^{26}$ en una revisión de 126 fracturas de escafoides tratadas mediante fijación percutánea asistida por artroscopia, solo tuvieron dos casos de fracaso de la consolidación. Thomsen y Falcone ${ }^{27}$ en 18 pacientes con buen resultado final, objetivaron una pérdida de fuerza inferior al 30\% respecto a la mano contralateral a los 6 meses de seguimiento. En 31 de los 33 pacientes presentados por 


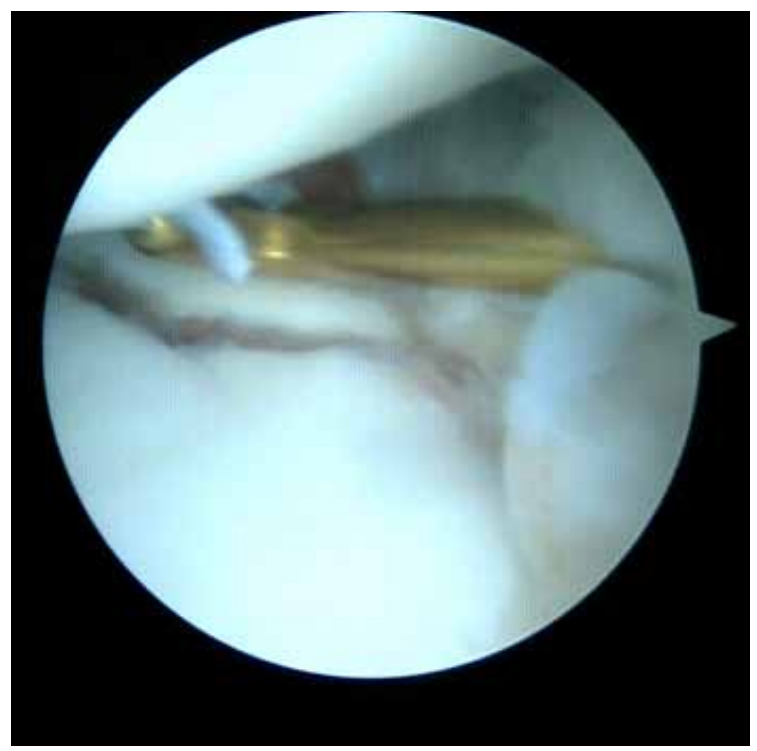

Figura 7. Reducción del fragmento distal con gancho palpador, introducido por el portal mediocarpiano radial.

Martinache y Mathoulin ${ }^{28}$, el arco de movilidad final era superior al $90 \%$ del lado contralateral.

La cirugía artroscópica en las fracturas de escafoides es una técnica exigente que requiere una curva de aprendizaje y que no está exenta de complicaciones, por lo que el cirujano debe iniciarse en ella una vez adquirida experiencia en otras técnicas más básicas de artroscopia de muñeca. Pueden originarse lesiones del aparato extensor, rotura de agujas guía y lesión de los nervios sensitivos en los portales artroscópicos.

\section{CONCLUSIÓN}

En conclusión, el uso de la artroscopia como coadyuvante en el tratamiento quirúrgico de las fracturas de escafoides, nos ofrece una visualización óptima del desplazamiento de la fractura, nos permite un diagnóstico preciso de las lesiones ligamentosas asociadas a estas fracturas incluso superando a la cirugía abierta por lo que se refiere a las lesiones del borde cubital del carpo, facilita la reducción de los fragmentos y permite una correcta monitorización de la colocación subcondral del tornillo de compresión en el polo proximal, todo ello utilizando una técnica mínimamente invasiva.

Es aconsejable su utilización en todas las fracturas inestables de escafoides y en aquellas no desplazadas o mínimamente desplazadas en las que se quiera indicar un protocolo de movilización precoz tras la osteosíntesis.

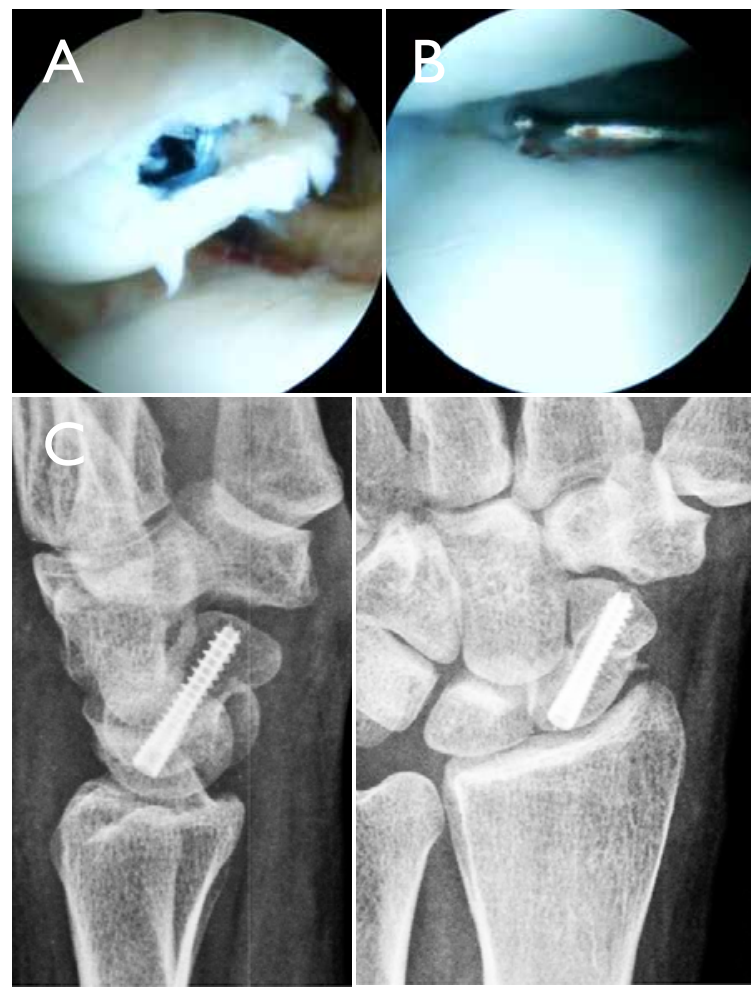

Figura 8. A. Comprobación de correcta colocación de tornillo desde portal radiocapiano 6R. B. Comprobación final de la reducción desde portal mediocarpiano cubital. C. Control radiológico.

\section{CONFLICTOS DE INTERESES}

Los autores declaran no tener conflictos de intereses.

\section{BIBLIOGRAFÍA}

I. Dias JJ, Wildin CJ, Bhowal B, Thompson JR. Should acute scaphoid fractures be fixed? A randomized controlled trial. J Bone Joint Surg Am. 2005;87( I0):2160-8.

2. Vinnars B, Pietreanu M, Bodestedt A, Ekenstam Fa, Gerdin B. Nonoperative compared with operative treatment of acute scaphoid fractures. A randomized clinical trial. J Bone Joint Surg Am. 2008;90(6): I 176-85.

3. Ibrahim T, Qureshi A, Sutton AJ, Dias JJ. Surgical versus nonsurgical treatment of acute minimally displaced and undisplaced scaphoid waist fractures: pairwise and network meta-analyses of randomized controlled trials. J Hand Surg Am. 2011;36:1759-1768. 
4. Bond CD, Shin AY, McBride MT, Dao KD. Percutaneous screw fixation or cast immobilization for nondisplaced scaphoid fractures. J Bone Joint Surg Am. 200।;83 (4):483-8.

5. Arora R, Gschwentner M, Krappinger D, Lutz M, Blauth M, Gabl M. Fixation of nondisplaced scaphoid fractures: making treatment cost effective. Prospective controlled trial. Arch Orthop Trauma Surg. 2007; I 27(I):39-46.

6. Buijze GA, Doornberg JN, Ham JS, Ring D, Bhandari M, Poolman RW. Surgical compared with conservative treatment for acute nondisplaced or minimally displaced scaphoid fractures: a systematic review and meta-analysis of randomized controlled trials. J Bone Joint Surg Am. 20 I 0;92(6): I 534-44.

7. Herbert $T$ J, Fisher W E: Management of the fractured scaphoid using a new bone screw. J Bone Joint Surg (Br) 1984;66( I): | |4-23.

8. Rettig ACm Weidenbener EJ, Gloyeske R ; Alternative management of midthird scaphoid fractures in the athlete. Am J Sports Med 1994;22(5):7 I I-4.

9. Trumble TE, Gilbert M, Murray IW, Smith J, Rafijah G, Mc- Callister WV: Displaced scaphoid fractures treated with open reduction and internal fixation with a cannulated screw. J Bone Joint Surg (Am) 2000;82:633-41.

10. Slade JF 3rd, Grauer JN, Mahoney JD. Arthroscopic reduction and percutaneous fixation of scaphoid fractures with a novel dorsal technique. Orthop Clin North Am. 200 I;32:247-6I.

II. Filan SI, Herbert T): Herbert screw fixation of scaphoid fractures.J Bone Joint Surg (Br) 1996;78B:5 19-29.

12. Whipple TL. Stabilization of the fractured scaphoid under arthroscopic control. Orthop Clin North Am 1995;26:749-54.

13. Taras JS, Sweet S, Shum W, Weiss LE, Bartolozzi A. Percutaneous and arthroscopic screw fixation of scaphoid fractures in the athlete. Hand Clin. 1999; 15(3):467-73.

14. Slade JF, Lozano-Calderón S, Merrell G, Ring D. Arthroscopic-assisted percutaneous reduction and screw fixation of displaced scaphoid fractures. J Hand Surg Eur 2008;33(3):350-4.

15 Geissler WB. Arthroscopic management of scaphoid fractures in athletes. Hand Clin. 2009;25(3):359-69.

16. Dias JJ, Singh HP. Displaced fracture of the waist of the scaphoid. J Bone Joint Surg Br. 201 1;93( I I): 1433-9.
17. Buijze GA, Jørgsholm P, Thomsen NO, Björkman A, Besjakov J, Ring D. Factors associated with arthroscopically determined scaphoid fracture displacement and instability. I Hand Surg Am. 2012;37(7): | 405- I0.

18. Buijze GA, Jørgsholm P,Thomsen NO, Bjorkman A, Besjakov J, Ring D. Diagnostic performance of radiographs and computed tomography for displacement and instability of acute scaphoid waist fractures.J Bone Joint Surg Am. 20 I2;94(21): 1967-74.

19. Mayfield JK, Johnson RP, Kilcoyne RK. Carpal dislocations: pathomechanics and progressive perilunar instability. J Hand Surg 1980; 5:226 -4l.

20. Braithwaite IJ, Jones WA. Scapho-lunate dissociation occurring with scaphoid fracture.J Hand Surg 1992; 17B:286 -8.

21. Shih JT, Lee HM, HouYT, Tan CM. Results of arthroscopic reduction and percutaneous fixation for acute displaced scaphoid fractures. Arthroscopy. 2005;2I (5):620-6.

22. Caloia MF, Gallino RN, Caloia H, Rivarola H. Incidence of ligamentous and other injuries associated with scaphoid fractures during arthroscopically assisted reduction and percutaneous fixation. Arthroscopy. 2008;24(7):754-9.

23. Jørgsholm P, Thomsen NO, Björkman A, Besjakov J, Abrahamsson SO. The incidence of intrinsic and extrinsic ligament injuries in scaphoid waist fractures. J Hand Surg Am. 20 10;35(3):368-74.

24 Meermans G, Verstreken F. Influence of screw design, sex, and approach in scaphoid fracture fixation. Clin Orthop Relat Res. 20 12;470(6): I 673-8I.

25. Geissler WB, Freeland AE, Savoie FH, McIntyre LW, Whipple TL. Intracarpal soft-tissue lesions associated with an intra-articular fracture of the distal end of the radius.J Bone Joint Surg 1996;78A:357-65.

26. Slade JF, Gillon T. Retrospective review of 234 scaphoid fractures and nonunions treated with arthroscopy for union and complications. Scand J Surg. 2008;97:280-9.

27. Thomsen L, Falcone MO. Lesions of the scapholunate ligament associated with minimally displaced or non-displaced fractures of the scaphoid waist. Which incidence?. Chir Main.2012;31:234-8.

28. Martinache $X$, Mathoulin C. Percutaneous fixation of scaphoid fractures with arthroscopic assistance. Chin Main. 2006;25:SI7I-7. 